\title{
Mechanisms Adjustable Design of One-side Sewing Device Apply in Textile Composites
}

\author{
Fulin Yao ${ }^{1, a^{*}}$,Liangyu $\mathrm{Li}^{1, \mathrm{~b}}$,Jianfeng Yue ${ }^{1, \mathrm{c}}$ \\ ${ }^{1}$ Advanced Mechatronics Equipment Technology Tianjin Area Major Laboratory, \\ Tianjin Polytechnic University, Tianjin 300387, China \\ ayaofulin111@163.com, 'liangyu1965@163.com, 'billyue163.com
}

Keywords: one-side stitching; suture stitch; adjustable design.

\begin{abstract}
One-side sewing technique can avoid the space limitation when processing the pre-shaped textile composites compare with the traditional sewing method. In order to adapt to the changes of parameters such as the thickness of the sewing material in the different operating environment, this article focus on adjustable design of mechanisms in one-side sewing device, utilize the method of changing the needle puncture angle and movement stroke of the needle-bar at the same time, realized the continuous adjustment of the suture parameters. Accordingly, analyzed and determined the stitching work space and the optimal parameter range of sewing material.
\end{abstract}

\section{Introduction}

One-side sewing technique is a new method of three-dimensional textile preform processing, which can avoid the space limitation when processing the pre-shaped textile composite compare with the traditional method. Studies have shown that the textile composites has a good layers performance and impact damage tolerance which use the method of one-side sewing technique[1-3]. Figure 1 shows the three-dimensional model of one-side sewing device. This paper introduces the mechanisms and work principle of one-side sewing device, and focus on adjustable design of mechanisms in one-side sewing device, utilize the method of changing the needle puncture angle and movement stroke of the needle-bar at the same time, realized the continuous adjustment of the suture parameters. Accordingly, analyzed and determined the optimal parameter range of the thickness about sewing material, and afford a theoretical basis for debugging and construct the expected suture stitch during the process.

\section{The basic work principle of the one-side sewing device}

The structure of one-side sewing device mainly consists of three parts, they are thread-leading, thread-hooking and thread-taking-up mechanism. The transmission of the mechanism is rely on the gears and synchronous toothed belt. Mechanisms schematic diagram shown in figure 1(b).

The forming process of stitch shown in figure 2. The leading-needle with sutures puncture the surface of the workpiece, and form a sewing-loop during the returning motion of the needle-bar. The hooking-needle hook the sewing-loop from lower surface of the workpiece and take back the sutures in the process of returning motion, and a work cycle is completed. By multiple cycles of reciprocating motion, interlocked chain stitches were formed which shown in figure 2 . In this process, the role of thread-taking-up mechanism is prefeeding and tighten up the sutures of the stitch which already formed, and new sutures are pulled out from the spool simultaneously in order to meet the thread consumption of the needles works in conjunction. 


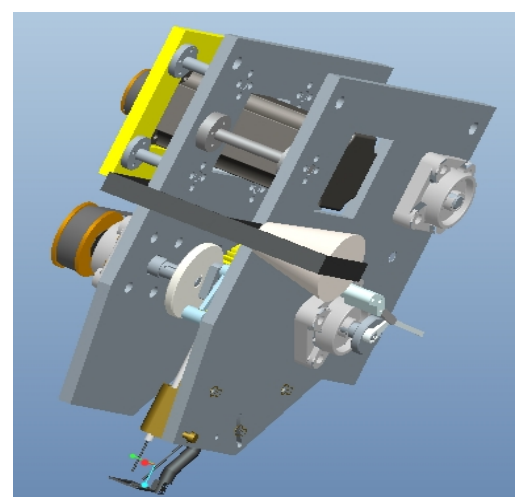

(a) three dimensional model of equipment

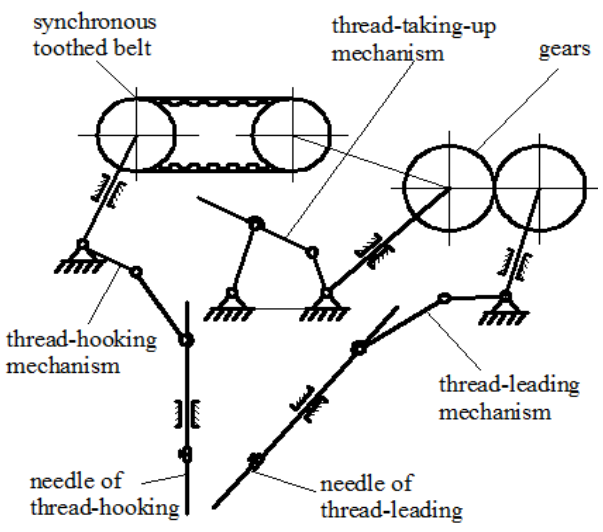

(b) draft of one-side sewing mechanism

Fig.1 Draft of one-side sewing mechanism

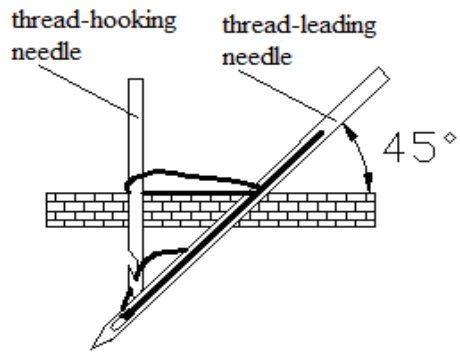

(a)forming process of stitch

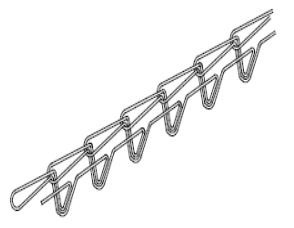

(b)chain stitch

Fig.2 Principle of the forming of seam

\section{Adjustable design of mechanisms based on the thickness of the sewing material}

Manipulate objects of one-side sewing device are mostly three-dimensional shape or profiled workpieces. For the work pierecs, the thickness of sewing material are different, and parameters of stitch length and seam width are also mutative in different circumstances. Because of the essential factors which impact sewing parameters is the change of work location during the cooperating work of the needles, (that the change in position of thread-loop formed on the leading-needle and hooking-needle hook the thread-loop), as only as utilize the method of changing the needle puncture angle and movement stroke of the needle-bar at the same time which shown in figure 3, the continuous adjustment of the suture parameters will be realized.

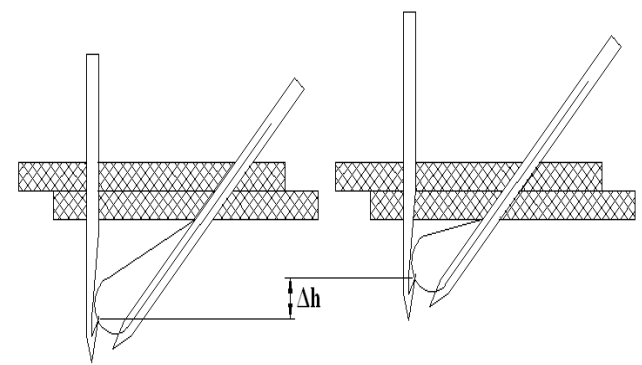

Fig. 3 the change of work location during the cooperating work of the needles

In order to achieve continuous adjustment of the puncture angle of needles relative to sewing material, the sliding bearing housing of thread-leading and thread-hooking mechanism has been renovation designed which play role in guiding the needle bar. As shown in figure 4, Make one end of the sliding bearing housing fixed, and another end can be changed in a certain range of angles. 
After rebuilding design, needles puncture angle of thread-leading and thread-hooking mechanism can be realized continuous adjustment in range of $30^{\circ}$ and $35^{\circ}$.
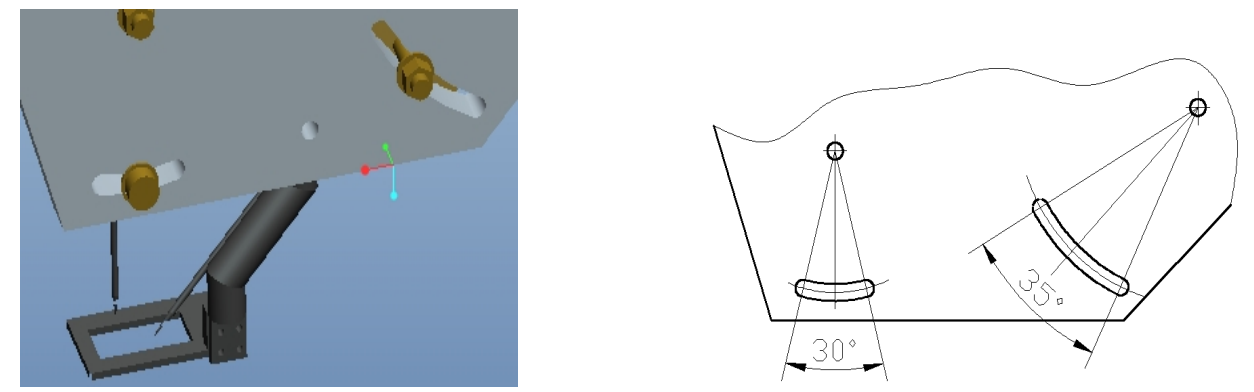

Fig.4 Angle adjustment of sliding bearing seat

On the other hand, for the purpose of changing the work location during the cooperating work of the needles, movement stroke of the needle-bar also need to be adjusted accordingly. In order to realize continuous adjustment of movement stroke of the needle-bar, the thread-leading and thread-hooking mechanism adopts the innovative design of double crank disk.

In thread-leading and thread-hooking mechanism, crank portions are respectively composed of two eccentric wheels with eccentric distance $\mathrm{e}_{1}=\mathrm{OO}_{1}$ and $\mathrm{e}_{2}=\mathrm{AO}_{1}$ which as shown in figure 5. By changing the relative circumferential position of two eccentric wheels(that change the relative position of point $\mathrm{A}, \mathrm{O}$ and $\mathrm{O}_{1}$ ), continuous adjustment of the length of the crank can be realized, and the slider has a different amount of displacement. Adjustment range of crank length is:

$$
\left\{\begin{array}{l}
l_{1}=O A_{M a x}=O O_{1}+A O_{1} \\
l_{2}=O A_{M i n}=O O_{1}-A O_{1}
\end{array}\right.
$$

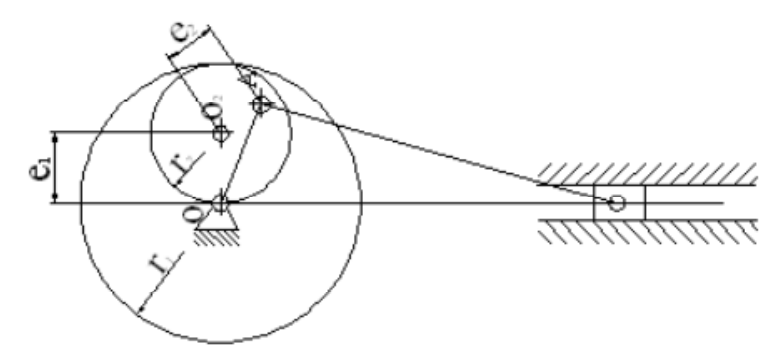

Fig.5 Adjustable design of thread-leading and thread-hooking mechanism

\section{Analysis of stitching work space}

In order to determine the parameters about the thickness range of workpiece in different operating environment, Take the suture needles of thread-leading and thread-hooking mechanism as research object, and established XOY coordinate system as shown in figure 6(a). Straight line $Y_{1} 、 \mathrm{Y}_{2}$ and $\mathrm{Y}_{3}$ 、 $\mathrm{Y}_{4}$ respectively expressed limit regulating position of needles puncture angle which ralative to surface of the workpiece. Arc $\mathrm{O}_{1} 、 \mathrm{O}_{2}$ and $\mathrm{O}_{3} 、 \mathrm{O}_{4}$ respectively expressed attainable maximum displacement amount of leading and hooking needles after continuous adjustment. The expression of angle and stroke adjustment range shown in table 1.

According to the formula in table 1, map out the operating space of leading and hooking needle at the state of work in conjunction. As shown in figure 6(b), the area of graphic displays is the achievable the maximum limit position of leading and hooking needles with parameter 
adjustment,and the region surrounded by point $\mathrm{A} 、 \mathrm{~B} 、 \mathrm{C}$ and $\mathrm{D}$ is the preferably shaped. Therefore, the distance between the straight line of $\mathrm{AB}$ and $\mathrm{CD}$ is the optimal thickness range of the workpiece, and the figure shows that the range of thickness in sewing operations is $20 \mathrm{~mm}$.

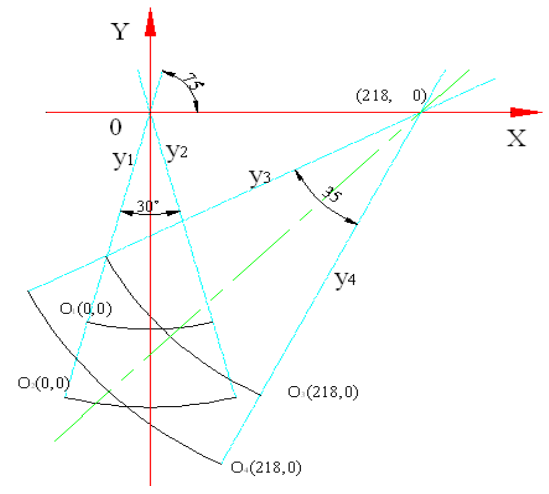

(a)needles coordinate system

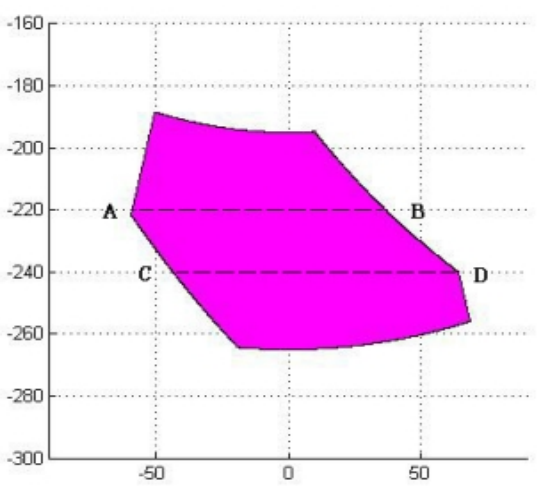

(b)stitching work space

Fig.6 Analysis of stitching work space

Tab.1 The expression of angle and stroke adjustment range

\begin{tabular}{c|c|c}
\hline & $\begin{array}{l}\text { Thread-leading } \\
\text { needle }\end{array}$ & Thread-hooking needle \\
\hline \multirow{2}{*}{ Angle adjustment range } & $\begin{array}{c}y_{1}=\tan 75^{\circ} x \\
y_{2}=-\tan 75^{\circ} x\end{array}$ & $\begin{array}{c}y_{3}=\tan 28^{\circ}(x-218) \\
y_{4}=\tan 63^{\circ}(x-218)\end{array}$ \\
\hline Stroke adjustment range & $x^{2}+y^{2}=195^{2}$ & $(x-218)^{2}+y^{2}=285^{2}$ \\
& $x^{2}+y^{2}=265^{2}$ & $(x-218)^{2}+y^{2}=355^{2}$ \\
\hline
\end{tabular}

\section{Conclusion}

This paper introduces the mechanisms and work principle of one-side sewing device, and focus on adjustable design of mechanisms in one-side sewing device, utilize the method of changing the needle puncture angle and movement stroke of the needle-bar at the same time, realized the continuous adjustment of the suture parameters. Accordingly, analyzed and determined the optimal parameter range of the thickness about sewing material. and afford a theoretical basis for debugging and construct the expected suture stitch during the process. At the present stage,one-side sewing device is in phase of structural design and theoretical research, adjustable design of mechanisms in one-side sewing can afford a theoretical basis for debugging and construct the expected suture stitch during the process.

\section{Acknowledgements}

This work is supported by The key technologies R \& D program of Tianjin(NO.15ZCZDGX00300).

\section{References}

[1] AI Tao, Wang Rumin. Progress of research on Kevlar stitched composite[J]. Material Review,2005,19(1):64-67.(in chinese)

[2] Drechsler. Latest developments in stitching and braiding technologies for textile performing[J].International SAMPE Symposium and Exhibition Proceedings, 2004, 49:2055-2067. 
[3] Herkt, ,Manuel.3D-reinforcement of composite T-joints by means of robot assisted stitching technology[J].International SAMPE Symposium and Exhibition Proceedings, 2006, 51:22-29. 